\title{
Exact Size-Dependent Elasticity Solution for Free Radial Vibration of Nano-Sphere
}

\author{
Yaser Mirzaei \\ Department of Mechanical Engineering, Damavand Branch, Islamic Azad University, Damavand, Iran.
}

(Received 7 June 2017; accepted 7 June 2018)

An exact size-dependent elasticity solution based on surface energy theory is used to investigate the free radial vibration behavior of a nanoscale sphere. The Gurtin-Murdoch surface elasticity model is employed to incorporate surface stress terms into pertinent boundary conditions. This leads to frequency equations involving spherical Bessel functions. Extensive numerical calculations have been carried out to illustrate the size effect of the nanosphere on the first and second dimensionless breathing natural frequencies. The numerical results describe the imperative influence of surface energy and radii ratio on the vibrational characteristic frequency of the nano-sphere. In particular, the surface energy is much more important when the inner radius is smaller than $50 \mathrm{~nm}$.

\section{INTRODUCTION}

The vibrational behavior of spherical shells has been extensively investigated in the literature for more than a century. Many of these studies are based on thin/thick shell theories or three dimensional elasticity theory. Many researchers have carried out vibrational analysis based on the linear equations of elasticity in order to find accurate natural frequencies of solid or hollow spheres. The most important works will briefly be reviewed here. The first known complete mathematical model for the vibration of an ideal isotropic elastic sphere was given by Lamb. ${ }^{1}$ Specifically, the problem of the free vibration of a sphere was reformulated and two basic types of free vibration was presented. The first class is torsional vibrations, or vibrations associated with the rotatory motions of the sphere, where there is no change in volume of the sphere and no radial displacement. The second class is spheroidal, pulsating or coupled bending-stretching, vibration which are related to the distortion of the elastic sphere due to vibrations in the radial direction. ${ }^{1}$ Most of the subsequent studies were based on the work of Lamb. Sato and Usami ${ }^{2}$ provided a detailed spectral analysis along with a comprehensive set of results for the frequencies of free vibration and their corresponding displacement field distributions within vibrating homogeneous elastic spheres. Subsequently Shah et al.3 studied the free vibrational behavior of hollow spheres by using an exact three-dimensional analysis to obtain a characteristic equation in terms of spherical Bessel functions of the first and second kind. Shah et al. ${ }^{3}$ gave numerical results for a wide range of thickness to radius ratios in graphical form. Hasheminezhad and Mirzaei ${ }^{4}$ developed an exact three-dimensional elasticity solution to describe the natural oscillations of a freely suspended, isotropic, and homogeneous elastic sphere with an eccentrically located inner spherical cavity analysis for describing both the natural and the extensive numerical calculations to illustrate the influence of cavity eccentricity on natural frequencies and mode shapes.
Recently, due to the increased application of nanoscale structures like the nano-beam, ${ }^{5}$ nano-bar, ${ }^{6}$ and nano-plate ${ }^{7}$ in technological systems, there has been an increase in demand for understanding the behavior of small-sized materials and structures. One of the procedures for studying the characteristics nano-sized materials and devices is "atomic simulation". 8 Another procedure is "size-depended elasticity theories". 5 In particular, several size-dependent elasticity theories are used to study very small-sized structures. One of the size-dependent elasticity theories is the "nonlocal elasticity theory." This theory was first offered by Eringen in the late 1960s and early 1970s. ${ }^{9-12}$ Wang and Varadan ${ }^{13}$ developed the nonlocal shell theory to investigate wave propagation in carbon nanotubes. Recently, Fazelzadeh and Ghavanloo ${ }^{14}$ employed the nonlocal elasticity theory to investigate the axisymmetric vibration of a fluid-filled spherical membrane shell. Also, Ghavanloo and Fazelzadeh ${ }^{15}$ used the nonlocal elasticity theory to achieve differential equations for the radial vibration of a nano-sphere. Following this, the characteristic equations for the radial vibration of a spherical shell were obtained and the variations of the frequencies with the nonlocal parameter, radii ratio and Poisson's ratio were demonstrated. Another well-known sizedependent elasticity theory is based on "surface energy." By increasing the ratio of surface area to volume, the surfaces and interfaces have considerable, or even dominant, influence in their deformation nature, performance, and reliability. ${ }^{16,17} \mathrm{~A}$ size-dependent elasticity theory based on surface energy was first documented by Gurtin and Murdoch ${ }^{18}$ to consider surface effects. Gurtin et al. ${ }^{19}$ proceeded to extend this work to incorporate the effects of interfaces as well. Shenoy ${ }^{20}$ used atomic calculations to achieve the surface elastic constants. Shenoy ${ }^{21}$ studied the deformation of some elementary nano-sized devices (e.g., beams, tubes, and plates) to compare the predictions of Gurtin's surface/interface elasticity theory via atomic simulations so reasonably well agreement between them is found. Hasheminezhad and Avazmohammadi ${ }^{22}$ investigated 
the diffraction of plane compressional/shear waves on nanosized inhomogeneity and the influence of surface effects on the dynamic stress concentration around the nano-inhomogeneity is shown. Moreover, He and Lilley ${ }^{23}$ studied the influence of the surface stress on the resonance frequencies of bending nanowires by using Euler-Bernoulli beam theory to obtain the natural frequencies of a micro-beam.

Finally, it should be noted that a basic and fundamental nano-problem was considered. Such analysis not only brings out the vibrational characteristics of the sphere at the nanoscale (fundamental motivations), such as, ${ }^{25-29}$ but can also provide reliable solutions for benchmarking purposes (technological motivations) in every field that deals with the wave propagation of a nano spherical particle, ${ }^{30}$ resonant ultrasound microscopy, ${ }^{31}$ nano-mechanical mass sensor. ${ }^{32}$ Thus, there are lot motivations to investigate the vibrational characteristics of nano spheres. Although the breathing natural frequency of a nano-sized spherical shell is obtained by using nonlocal elasticity theory, ${ }^{15}$ as far as the author knows, the investigation of the surface effects on breathing natural frequency of nanosized sphere is still lacking. The classical elasticity theory, in conjunction with the Gurtin-Murdoch theory, is used to determine the exact size-dependent elasticity solution for the breathing vibrational analysis of a nano-sized sphere. The influence of surface energy on vibration frequencies is examined. The results of this investigation may be useful in the study of the dynamic behavior of spherical nanoparticles, metal nano-shells and spherical core-shell nanoparticles.

\section{FORMULATION}

\subsection{Basic Field Equations}

The elastic material of the nano-sphere (Fig. 1) under consideration was assumed to be linear, homogeneous, and isotropic. In the absence of body forces, the displacement field was governed by the classical Navier's equation ${ }^{24}$

$$
\rho \frac{\partial^{2} \mathbf{u}}{\partial t^{2}}=\mu \nabla^{2} \mathbf{u}+(\lambda+\mu) \nabla(\nabla \cdot \mathbf{u}) .
$$

Subjected to appropriate boundary conditions. Here, $\rho$ denoted the elastic material density, $\lambda, \mu$ Lame constants and $\mathbf{u}$ denoted the vector displacement that was decomposed as the sum of an irrotational part $\mathbf{u}^{\prime}$. This corresponded with a compressional wave and a solenoidal part $\mathbf{u}^{\prime \prime}$, which corresponded with a shear wave: ${ }^{3}$

$$
\mathbf{u}=\mathbf{u}^{\prime}+\mathbf{u}^{\prime \prime}
$$

where $\nabla \cdot \mathbf{u}^{\prime \prime}=\mathbf{0}, \nabla \times \mathbf{u}^{\prime}=\mathbf{0}$. The above decomposition enables one to separate the dynamic equation of motion (1) into the decoupled vector Helmholtz equations.

$$
\begin{gathered}
\nabla^{2} \mathbf{u}^{\prime}+\alpha^{2} \mathbf{u}^{\prime}=0 ; \\
\nabla^{2} \mathbf{u}^{\prime \prime}+\beta^{2} \mathbf{u}^{\prime \prime}=0
\end{gathered}
$$

where $\nabla^{2}$ denoted the Laplacian, $\alpha^{2}=\omega^{2} / c_{p}^{2}, \beta^{2}=\omega^{2} / c_{s}^{2}$, $c_{p}^{2}=(\lambda+2 \mu) / \rho$ and $c_{s}^{2}=\mu / \rho$ were the propagation velocities of compressional and shear waves in the elastic medium,

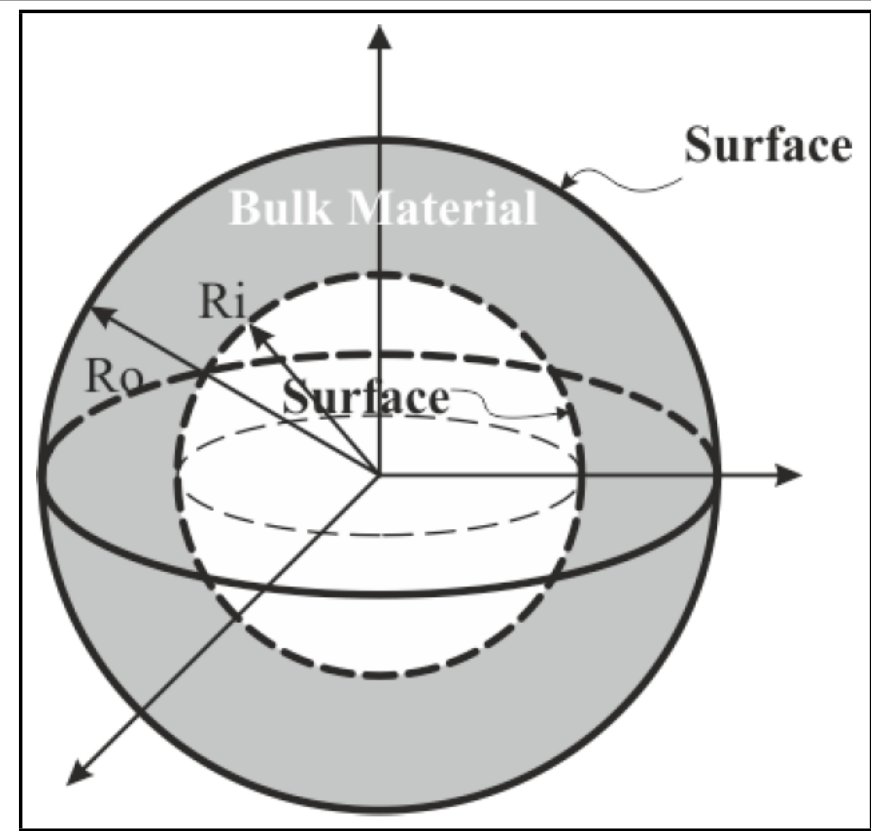

Figure 1. Nano-sphere

respectively. For this study, radial vibration that corresponded with a compressional wave was considered then $\mathbf{u}^{\prime \prime}=\mathbf{0}$. The solutions of the vector Helmholtz equations of irrotational part of displacement were written as

$$
\mathbf{u}^{\prime}=\nabla \varphi
$$

where the displacement potential $\varphi$ satisfy the scalar Helmholtz equations:

$$
\left(\nabla^{2}+\alpha^{2}\right) \varphi=0
$$

\subsection{Basic Surface Equations}

In the surface elasticity theory, a surface was considered as just a thin layer ideally adhered to the core bulk material without slipping. Additionally, the elastic constants of the surface layer were different from those of its bulk component. The equilibrium and constitutive equations in the bulk of the solid were the same as those in the traditional elasticity theory. However, the surface stress terms incorporate the pertinent boundary conditions ${ }^{18,19}$ The equilibrium equations on the surface were expressed as

$$
t_{\alpha}+\sigma_{\beta \alpha, \beta}^{s}=0, \quad\left\langle\sigma_{\beta \alpha}\right\rangle n_{i} n_{j}=\sigma_{\beta \alpha}^{s} \kappa_{\alpha \beta} ;
$$

where $n_{i}$ denoted the normal vector of the surface, $t_{\alpha}$ was the tangential component of the traction $t_{i}=\left\langle\sigma_{i j}\right\rangle n_{j}$ in the $x_{\alpha}$ direction. $\left\langle\sigma_{i j}\right\rangle$ was the jump of the bulk stress tensor across the surface. $\sigma_{\alpha \beta}^{s}$ was the surface stress tensor and $\kappa_{\alpha \beta}$ denoted the curvature of the surface. For a curved surface, the surface stresses and the surface curvature played a dominant role in the surface equilibrium conditions. Therefore, the surface inertia forces were neglected.

For an isotropic surface, the surface stresses were given: ${ }^{13}$

$$
\sigma_{\alpha \beta}^{(s)}=2 \mu^{s} \varepsilon_{\alpha \beta}^{s}+\lambda^{s} \varepsilon_{\gamma \gamma}^{s} \delta_{\alpha \beta} ;
$$

where $\delta_{\alpha \beta}$ was the Kronecker delta, $\varepsilon_{\alpha \beta}^{s}$ was the surface strains, $\mu^{s}$ and $\lambda^{s}$ were surface elastic constants. 


\subsection{Field Expansions and Boundary Conditions}

The nano-sphere, with inner and outer radii $R_{i}$ and $R_{o}$, were considered respectively. Due to radial vibration analysis, the displacement field was only expressed as a function of the $r$ coordinate. The solution of the scalar Helmholtz equation (5), which lead to the field expansion of compressional waves, was written as: ${ }^{24}$

$$
\varphi(r, \omega)=a(\omega) j_{0}(\alpha r)+b(\omega) y_{0}(\alpha r) ;
$$

where $a(\omega)$ through $b(\omega)$ were unknown modal coefficients, $j_{0}$ and $y_{0}$ were the spherical Bessel functions of the first and second kind, respectively. By substituting equation (8) into (4), the relevant displacement component was written as

$u_{r}(r, \omega)=a(\omega) V^{(1)}+b(\omega) V^{(2)}, u_{\phi}(r, \omega)=u_{\theta}(r, \omega)=0 ;$

in which

$$
V^{(i)}(r, \omega)=-\alpha \ell_{1}^{(i)}(\alpha r)
$$

where $i=1,2, \ell_{n}^{(\mathrm{i})}=\left\{\begin{array}{ll}j_{n} & (\mathrm{i}=1) \\ y_{n} & (\mathrm{i}=2)\end{array}, j_{n}\right.$ and $y_{n}$ were the spherical Bessel functions. Also, the stress tensor may be written in terms of the displacement vector as

$$
\sigma=\lambda(\nabla . \mathbf{u}) \mathbf{I}+\mu(\nabla . \mathbf{u}+\mathbf{u} \nabla)
$$

where I was the unit tensor. Therefore stress components can be respectively written as

$$
\begin{gathered}
\sigma_{r r}(r, \omega)=a(\omega) T_{1}^{(1)}+b(\omega) T_{1}^{(2)} ; \\
\sigma_{\theta \theta}(\theta, \omega)=\sigma_{\phi \phi}(\theta, \omega)=a(\omega) T_{2}^{(1)}+b(\omega) T_{2}^{(2)} ;
\end{gathered}
$$

in which

$$
\begin{gathered}
T_{1}^{(i)}(r, \omega)=\frac{2 \mu}{r}\left[-\frac{r \beta^{2}}{2} \ell_{0}^{(i)}(\alpha r)+2 \alpha \ell_{1}^{(i)}(\alpha r)\right] \\
T_{2}^{(i)}(r, \omega)=\frac{2 \mu}{r^{2}} \ell_{0}^{(i)}(\alpha r) .
\end{gathered}
$$

Surface equilibrium equations were taken into account in order to derive the pertinent non-classical boundary conditions in the spherical coordinate system as follow ${ }^{13}$

$\sigma_{r r}\left(\mathrm{R}_{\mathrm{i}}, \omega\right)=\frac{\left(\sigma_{\theta \theta}^{(s)}\left(\mathrm{R}_{\mathrm{i}}, \omega\right)+\sigma_{\phi \phi}^{(s)}\left(\mathrm{R}_{\mathrm{i}}, \omega\right)\right)}{\mathrm{R}_{\mathrm{i}}}=\frac{2 \sigma_{\theta \theta}^{(s)}\left(\mathrm{R}_{\mathrm{i}}, \omega\right)}{\mathrm{R}_{\mathrm{i}}}$,
$\sigma_{r r}\left(\mathrm{R}_{\mathrm{o}}, \omega\right)=\frac{\left(\sigma_{\theta \theta}^{(s)}\left(\mathrm{R}_{\mathrm{o}}, \omega\right)+\sigma_{\phi \phi}^{(s)}\left(\mathrm{R}_{\mathrm{o}}, \omega\right)\right)}{\mathrm{R}_{\mathrm{o}}}=\frac{2 \sigma_{\theta \theta}^{(s)}\left(\mathrm{R}_{\mathrm{o}}, \omega\right)}{\mathrm{R}_{\mathrm{o}}}$,

where $\sigma_{\theta \theta}^{(s)}(\mathrm{r}, \omega)$ was the surface stress at the surface. In fact, deriving surface stresses was necessary for determining surface strain. The surface strain, in terms of stress components in bulk material on the spherical interface, was written as: ${ }^{13}$

$$
\begin{aligned}
\varepsilon_{\theta \theta}^{(s)} & =\frac{1}{E}\left(\sigma_{\theta \theta}-\nu \sigma_{r r}-\nu \sigma_{\phi \phi}\right)=\frac{1}{E}\left((1-\nu) \sigma_{\theta \theta}-\nu \sigma_{r r}\right) \\
\varepsilon_{\phi \phi}^{(s)} & =\frac{1}{E}\left(\sigma_{\phi \phi}-\nu \sigma_{r r}-\nu \sigma_{\theta \theta}\right)=\frac{1}{E}\left((1-\nu) \sigma_{\theta \theta}-\nu \sigma_{r r}\right) .
\end{aligned}
$$

By substituting equation (10) into equation (12), the relevant surface strain components were written as

$$
\varepsilon_{\theta \theta}^{(s)}=\varepsilon_{\phi \phi}^{(s)}=\frac{1}{E}\left[a(\omega) S^{(1)}+b(\omega) S^{(2)}\right] ;
$$

in which

$$
S^{(i)}=\left((1-\nu) T_{2}^{(i)}-\nu T_{1}^{(i)}\right) .
$$

By substituting the above surface strain equation (13) into equation (7), the surface stress was written as:

$$
\sigma_{\theta \theta}^{(s)}=\sigma_{\phi \phi}^{(s)}=\frac{2\left(\mu^{s}+\lambda^{s}\right)}{E}\left[a(\omega) S^{(1)}+b(\omega) S^{(2)}\right] .
$$

Substituting the surface stresses components (14) into the nonclassical boundary conditions (11), yielded the following two equations:

$$
\begin{array}{r}
a(\omega)\left(T_{1}^{(1)}-k_{1} S^{(1)}\right)+b(\omega)\left(T_{1}^{(2)}-k_{1} S^{(2)}\right)=0 ; \\
\text { at } r=\mathrm{R}_{\mathrm{i}} ; \\
a(\omega)\left(T_{1}^{(1)}-k_{2} S^{(1)}\right)+b(\omega)\left(T_{1}^{(2)}-k_{2} S^{(2)}\right)=0 ; \\
\text { at } r=\mathrm{R}_{\mathrm{o}} ;
\end{array}
$$

where $k_{1}=\frac{4\left(\mu^{s}+\lambda^{s}\right)}{E R_{i}}$ and $k_{2}=\frac{4\left(\mu^{s}+\lambda^{s}\right)}{E R_{o}}$. The linear system of equations (15) were truncated into the matrix form:

$$
[\mathrm{M}][\mathrm{C}]=0
$$

where $\mathrm{C}=[a(\omega), b(\omega)]^{T}$ denoted the modal vector multiplied by $\mathrm{M}, 2 \times 2$ square matrix that contained frequencydependent parameters. By setting the determinant of the square matrix equal to zero, the characteristic equation of the system was obtained. This lead to the determination of the natural frequencies. Next, we consider some numerical examples.

\section{NUMERICAL RESULTS}

In order to illustrate the nature and general behavior of the solution and the influence of the surface stress on the vibrational performance of the nano-sphere, we considered a number of numerical examples in this section. A Mathematica code was constructed for the numerical calculation of the normalized natural frequencies $\omega /\left(c_{p} \mathrm{R}_{\mathrm{i}}\right)$ as a function of the dimensionless radii ratio parameter $R_{o} / R_{i}$ and inner radius $R_{i}$. In particular, a simple and very efficient root finding technique, which was based on the bisection approach, is employed to determine the roots of the characteristic equations. To this end, a nano-sphere with selected radii ratios is considered to be made of isotropic aluminum with the physical properties: $\rho=2700 \mathrm{~kg} / \mathrm{m}^{3}, \lambda=52.0 \times 10^{9} \mathrm{~N} / \mathrm{m}^{2}, \mu=34.7 \times 10^{9} \mathrm{~N} / \mathrm{m}^{2}$. Two different sets of surface properties corresponding to the crystallographic directions [100] (denoted by "SA") and [111] (denoted by "SB") in aluminum are used in the calculation. The corresponding elastic constants are given as: ${ }^{5}$

Surface A "SA": $E^{s}=-8.95(\mathrm{~N} / \mathrm{m})$,

Surface B "SB": $E^{s}=6.08(\mathrm{~N} / \mathrm{m})$. 


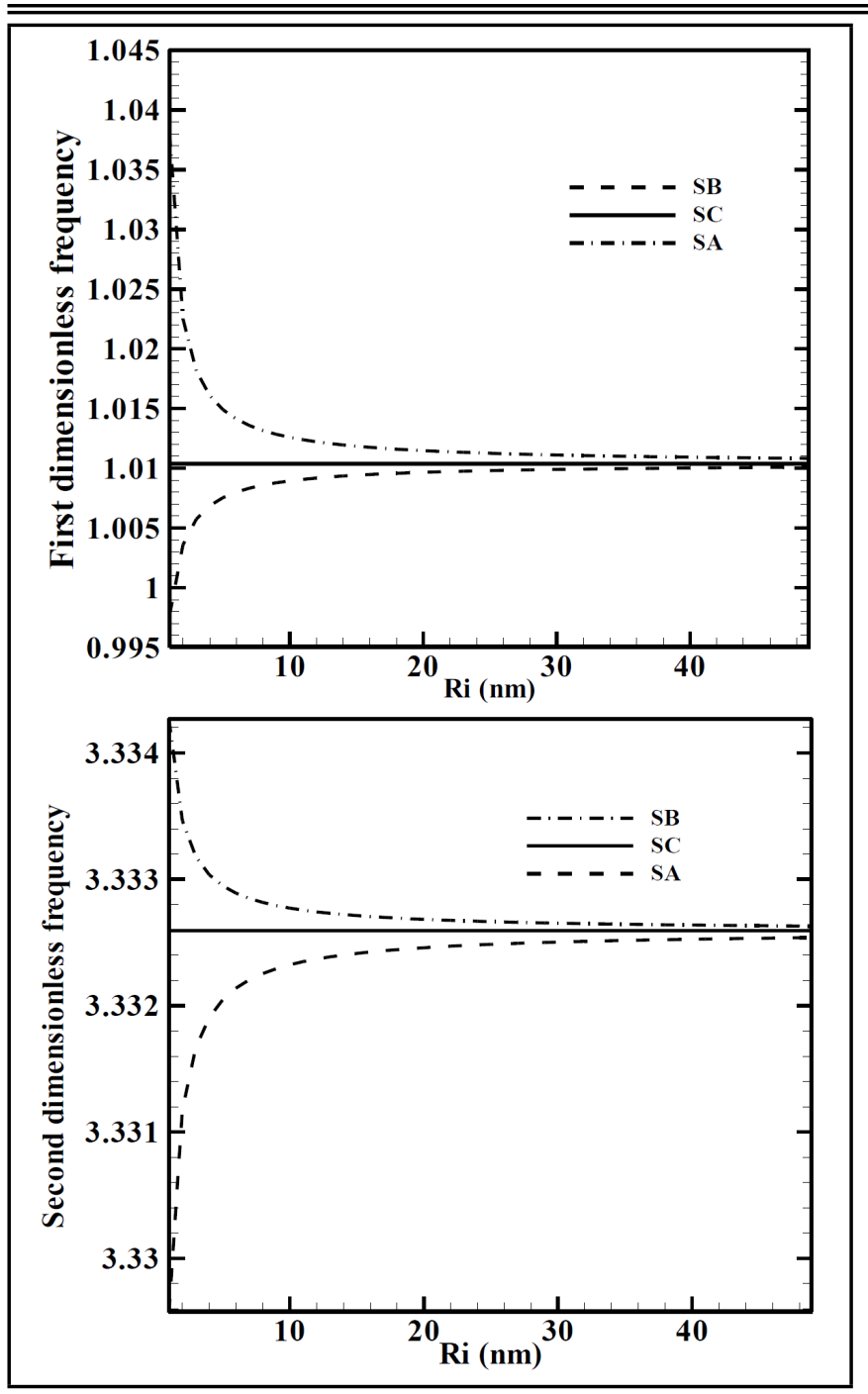

Figure 2. Variation of dimensionless radial natural frequencies versus inner radius of nano-sphere for radii ratio $R_{o} / R_{i}=2$ and selected surface type.

Classical elasticity theory, in which surface effects don't appear or $E^{s}=0(\mathrm{~N} / \mathrm{m})$, are denoted by Surface C "SC".

Figures 2 through 4 display the variation of the first two dimensionless radial natural frequencies $\omega /\left(c_{p} R_{i}\right)$ versus the inner radius of the nano-sphere for the selected inner-outer radius ratios of $\mathrm{R}_{\mathrm{o}} / \mathrm{R}_{\mathrm{i}}=1.1,1.5,2$. These figures included the results associated with "SA", "SB" and "SC" surfaces. The modal spectrums of nano-spheres exhibited very unique characteristics.

Regardless of the radii ratio, the dimensionless radial natural frequencies associated with $\mathrm{SA}, \mathrm{SB}$, and $\mathrm{SC}$ surfaces are the same as the inner radius of the nano-sphere, which is more than $50 \mathrm{~nm}$. While the inner radius of the nano-sphere decreases, the dimensionless radial natural frequencies associated with the SA, SB, and SC surfaces are split into three distinct eigen-frequencies. This frequency divergence increases with the decrease of the inner radius of the nano-sphere.

It can be observed that the surface energy has a more evident influence on dimensionless natural frequencies when innerouter radius ratios are increased. Also, while the absolute value for $E^{s}$ of "SA" is greater than that of "SB", the frequency divergence associated with "SA" from the classical solution is
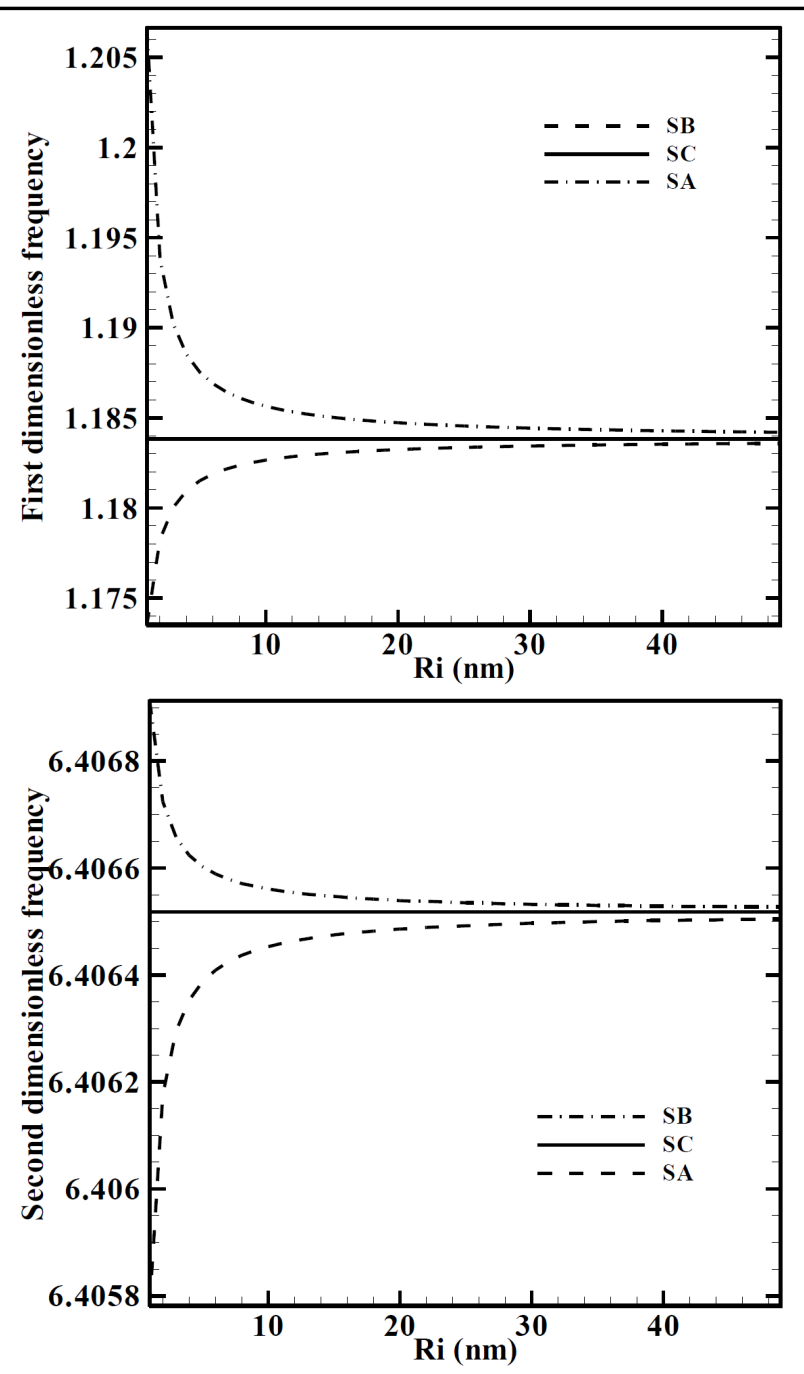

Figure 3. Variation of dimensionless radial natural frequencies versus inner radius of nano-sphere for radii ratio $R_{o} / R_{i}=1.5$ and selected surface type.

more than those associated with "SB". Finally, the frequencies associated with the SC surface are the same as the classical elasticity theory that was calculated by $\mathrm{Shah}^{3}$ and Sato. ${ }^{2}$

\section{CONCLUSIONS}

The Murdoch size-dependent elasticity theory is employed to study the size effect on free radial vibration behavior of a nanoscale sphere. The surface energy inflicts different effects on vibrational characteristics depending on the radii ratio and the size of the inner radius of problem. In particular, the influence is more important when the inner radius is smaller than $50 \mathrm{~nm}$. Two different sets of surface properties corresponding with the crystallographic directions [100] (denoted by "surface A") and [111] (denoted by "surface B") in aluminum are employed. Their results can been compared with Surface C (SC), which refers to the classical elasticity theory. Regardless of radii ratio, when the inner radius of the nano-sphere is more than $50 \mathrm{~nm}$, the frequencies associated with SA, SB, SC surfaces are the same. While the inner radius of the nano-sphere decreases, dimensionless radial natural frequencies associated with SA, SB, SC surfaces are gradually split into three distinct eigen-frequencies. Also, $E^{s}$. It can be observed that increas- 


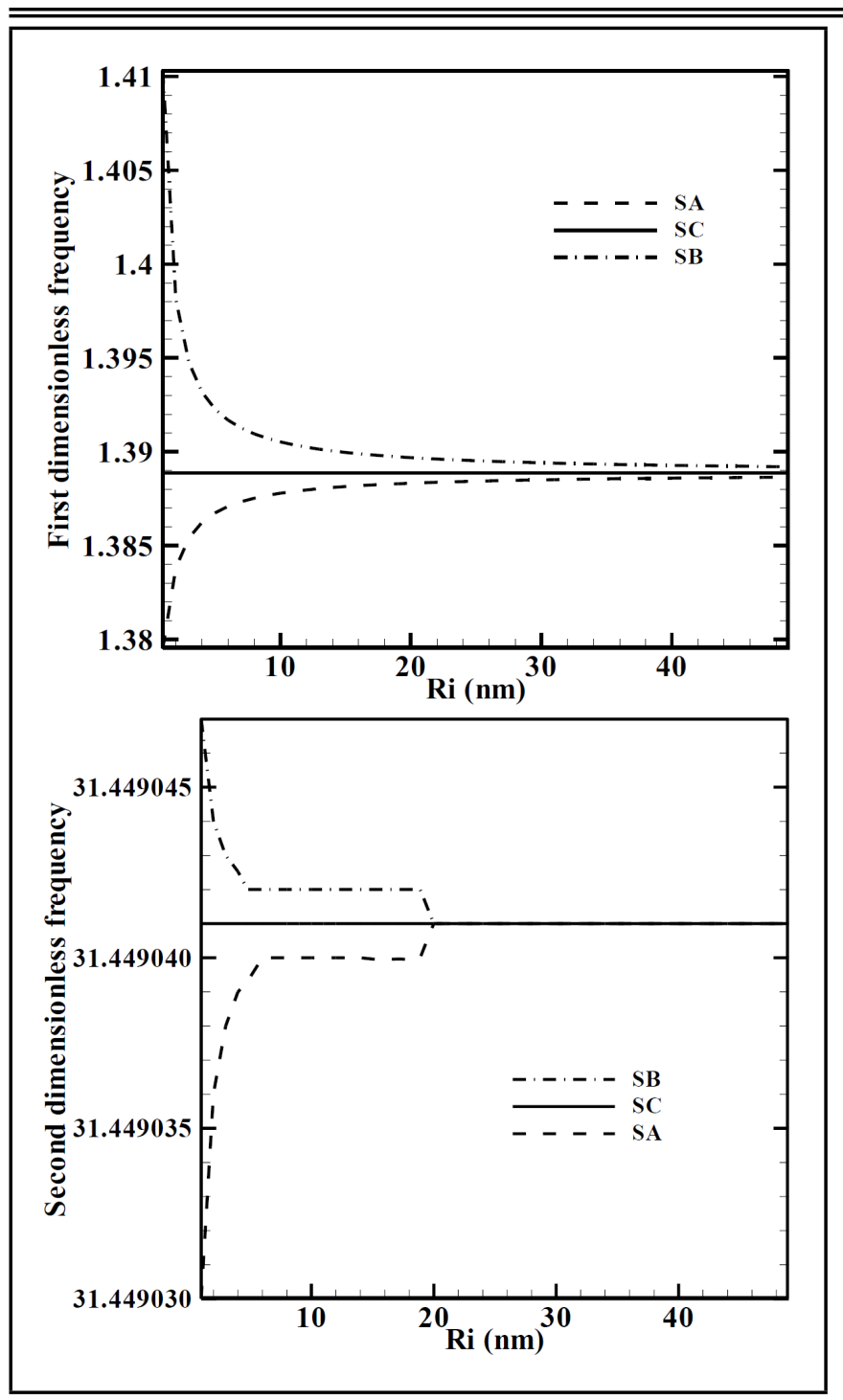

Figure 4. Variation of dimensionless radial natural frequencies versus inner radius of nano-sphere for radii ratio $R_{o} / R_{i}=1.1$ and selected surface type.

ing inner-outer radius ratios lead to an increase in the influence of the surface energy of natural frequencies.

\section{ACKNOWLEDGEMENTS}

The support from the AZAD UNIVERSITY OF DAMAVAND is acknowledged.

\section{REFERENCES}

1 Lamb, H. On the vibrations of an elastic sphere, Proceedings London Mathematical Society, 13, 189-212, (1882).

2 Sato, Y. and Usami, T. Basic study on the oscillation of a homogeneous elastic sphere; part II, distribution of displacement, Geophysics Magazine, 31, 25-47, (1962).

3 Shah, A. H., Ramakrishnan, C. V. and Datta, S. K. Three dimensional and shell theory analysis of elastic waves in a hollow sphere, Part I. Analytical foundation, Journal of Applied Mechanics, 36, 431-439, (1969). https://dx.doi.org/10.1115/1.3564698
${ }^{4}$ Hasheminejad, Seyyed M. and Mirzaei, Yaser. Exact 3D elasticity solution for free vibrations of an eccentric hollow sphere, Journal of Sound and Vibration, 330, 229-244, (2011). https://dx.doi.org/10.1016/j.jsv.2010.08.011

5 Eskandari, J., Mirzaei, Yaser, Gheghlaghi, B. and Avazmohamadi, R. Size-dependent free vibration analysis of infinite nanotubes using elasticity theory, Journal of Mechanics of Materials and Structures, 7, 137-144, (2012). https://dx.doi.org/10.2140/jomms.2012.7.137

6 Kiani, Keivan. Free dynamic analysis of functionally graded tapered nano-rods via a newly developed nonlocal surface energy-based integro-differential model, Composite Structures, 139, 151-166, (2016). https://dx.doi.org/10.1016/j.compstruct.2015.11.059

7 Ilkhani, M. R., Bahrami, A. and Hosseini-Hashemi, S. H. Free vibrations of thin rectangular nanoplates using wave propagation approach, Applied Mathematical Modelling, 40, 1287-1299, (2016). https://dx.doi.org/10.1016/j.apm.2015.06.032

8 Zhou, L. G. and Huang, H. C. Are surfaces elastically softer or stiffer?, Applied Physics Letter, 84, 1940-2, (2004). https://dx.doi.org/10.1063/1.1682698

9 Eringen, A. C. Nonlocal polar elastic continua, International Journal of Engineering and Science, 10, 1-16, (1972). https://dx.doi.org/10.1016/0020-7225(72)90070-5

10 Eringen, A. C. Linear theory of nonlocal elasticity and dispersion of plane waves, International Journal of Engineering and Science, 10, 425-435, (1972). https://dx.doi.org/10.1016/0020-7225(72)90050-X

11 Eringen, A. C. On differential equations of nonlocal elasticity and solutions of screw dislocation and surface waves, Journal of Applied Physics, 54, 4703-4710, (1983). https://dx.doi.org/10.1063/1.332803

12 Eringen, A. C. and Edelen, D. G. B. On nonlocal elasticity, International Journal of Engineering and Science, 10, 233-248, (1972). https://dx.doi.org/10.1016/00207225(72)90039-0

13 Wang, G. F., Feng, X. Q. and Yu, S. W. Interface effects on the diffraction of plane compressional waves by a nanosized spherical inclusion, Journal of Applied Physics, 102, 0435331-6, (2007). https://dx.doi.org/10.1063/1.2775217

14 Fazelzadeh, S. A. and Ghavanloo, E. Coupled axisymmetric vibration of nonlocal fluid-filled closed spherical membrane shell, Acta Mechanica, 223, 2011-2020, (2012). https://dx.doi.org/10.1007/s00707-012-0692-2 
15 Ghavanloo, E. and Fazelzadeh, S. A. Nonlocal elasticity theory for radial vibration of nanoscale spherical shells, $E$ ropean Journal of Mechanics A/Solids, 41, 37-42, (2013). https://dx.doi.org/10.1016/j.euromechsol.2013.02.003

16 Wong, E., Sheehan, P. E. and Lieber, C. M. Nano-beam Mechanics: Elasticity, Strength, and Toughness of Nanorods and Nanotubes, Science, 277, 1971-1975, (1971). https://dx.doi.org/10.1126/science.277.5334.1971

17 Cuenot, S., Fretigny, C., Champagne, S. D. and Nysten, B. Surface tension effect on the mechanical properties of nanomaterials measured by atomic force microscopy, Phys. Rev. B, 69, 165410-5, (2004). https://dx.doi.org/10.1103/PhysRevB.69.165410

${ }^{18}$ Gurtin, M. E. and Murdoch, A. I. A continuum theory of elastic material surfaces, Arch. Ration. Mech. Anal, 57, 291-323, (1975). https://dx.doi.org/10.1007/BF00261375

${ }^{19}$ Gurtin, M. E., Weissmuller, J. and Larche, F. A general theory of curved deformable interfaces in solids at equilibrium, Philos. Mag. A, 78, 1093-1109, (1998). https://dx.doi.org/10.1080/01418619808239977

${ }^{20}$ Shenoy, V. B. Atomistic calculations of elastic properties of metallic fcc crystal surfaces, Physical Review B, 71, 094104-11, (2005). https://dx.doi.org/10.1103/PhysRevB.71.094104

${ }^{21}$ Shenoy, V. B. Size-dependent rigidities of nanosized torsional elements, International Journal of Solids and Structures, 39, 4039-4052, (2002). https://dx.doi.org/10.1016/S0020-7683(02)00261-5

${ }^{22}$ Hasheminejad, S. M. and Avazmohammadi, R. Size-dependent effective dynamic properties of unidirectional nanocomposites with interface energy effects, Compo. Sci. Tech, 69, 2538-2546, (2009).https://dx.doi.org/10.1016/j.compscitech.2009.07.007

${ }^{23} \mathrm{He}$, J. and Lilley, C. M. Surface stress effect on bending resonance of nanowires with different boundary conditions, Appl. Phys. Lett, 93, 263108-3, (2008). https://dx.doi.org/10.1063/1.3050108

${ }^{24}$ Pao, Y. H. and Mow, C. C. Diffraction of Elastic Waves and Dynamics Stress Concentration, Crane Russak, New York (1973).
25 Duval, E. Boukenter, A. and Champagnon, B. Vibration Eigenmodes and Size of Microcrystallites in Glass: Observation by Very-Low-Frequency Raman Scattering, Physical Review Letter, 56, 2052, (1986). https://dx.doi.org/10.1103/PhysRevLett.56.2052

26 Duval, E. Boukenter, A. and Champagnon, B. Electron-phonon coupling dynamics in very small (between 2 and $8 \mathrm{~nm}$ diameter) $\mathrm{Au}$ nanoparticles, The Journal of Chemical Physics, 112, 8613, (2000). https://dx.doi.org/10.1063/1.481167

27 Nisoli, M. De Silvestri, S. Cavalleri, A. Malvezzi, A. M. Stella, A. Lanzani, G. Cheyssac, P. and Kofman, R. Coherent acoustic oscillations in metallic nanoparticles generated with femtosecond optical pulses, Physical Review B, 55, 13424-13427, (1997). https://dx.doi.org/10.1103/PhysRevB.55.R13424

${ }^{28}$ Sauceda, H. E. Mongin, D. Maioli, P. Crut, A. Pellarin, M. Del Fatti, N. Vallée, F. and Garzn I. L. Vibrational properties of metal nanoparticles: atomistic simulation and comparison with time-resolved investigation, The Journal of Chemical Physics C, 116, 25147-25156,(2012). https://dx.doi.org/10.1021/jp309499t

${ }^{29}$ Hosseini-Hashemi, Sh. H. Sharifpour and F. Ilkhani, M. R. On the free vibrations of size-dependent closed micro/nano-spherical shell based on the modified couple stress theory, International Journal of Mechanical Sciences, 115-116, 501-515, (2016). https://dx.doi.org/10.1016/j.ijmecsci.2016.07.007

30 Voisin, C. Christofilos, D. Del Fatti, N. and Vallée, F. Environment effect on the acoustic vibration of metal nanoparticles, Physica B: Condensed Matter, 316-317, 89-94,(2002). https://dx.doi.org/10.1016/S09214526(02)00430-1

31 Tian, J. Ogi, H. and Hirao, M. Vibration analysis of an elastic-sphere oscillator contacting semi-infinite viscoelastic solids in resonant ultrasound microscopy, Journal of applied physics, 95, 8366-8374, (2004). https://dx.doi.org/10.1063/1.1737472

32 Natsuki, T. Shi, J. X. and Ni, Q. Vibration analysis of nanomechanical mass sensor using double-layered graphene sheets resonators, Journal of Applied Physics, 114, 094307, (2013). https://dx.doi.org/10.1063/1.4820522 